Commun. Korean Math. Soc. 27 (2012), No. 3, pp. 537-545

http://dx.doi.org/10.4134/CKMS.2012.27.3.537

\title{
SYNDETIC SEQUENCES AND DYNAMICS OF OPERATORS
}

\author{
Hamid Rezaei
}

\begin{abstract}
In the present paper, we show that a continuous linear operator $T$ on a Frechet space satisfies the Hypercyclic Criterion with respect to a syndetic sequence must satisfy the Kitai Criterion. On the other hand, an operator, hereditarily hypercyclic with respect to a syndetic sequence must be mixing. We also construct weighted shift operators satisfying the Hypercyclicity Criterion which do not satisfy the Kitai Criterion. In other words, hereditarily hypercyclic operators without being mixing.
\end{abstract}

\section{Introduction}

Let $X$ denote a separable infinite dimensional Frechet space and $L(X)$ stands for the space of continuous linear operators on $X$. The operator $T$ in $L(X)$ is said to be hypercyclic when there exists a vector $x$ in $X$ such that its orbit under $T$, i.e., $\operatorname{orb}(T, x)=\left\{T^{n} x: n \in \mathbb{N}\right\}$, is dense in $X$. The study of hypercyclic operators on a Banach space was initiated in 1969 when Rolewicz proved that any multiple $\lambda B,|\lambda|>1$, of the standard backward shift $B$ on $\ell^{p}, 1 \leq p<+\infty$, is hypercyclic [12]. It is interesting to know what type of operators can actually be hypercyclic: Backward and bilateral shifts [12, 15], translation and differentiation operators $[4,8]$, adjoint of multiplication operators [8], composition operators [4] and weighted composition operators [11, 17]. For a complete survey of hypercyclicity, see book [1].

Kitai [9] stated in particular, a simple, useful and general criterion for hypercyclicity of operators which was isolated in a restricted form. Then it was independently rediscovered by R. Gethner and J. H. Shapiro in a general form and with a weakened assumptions [6]. This criterion referred to as the Hypercyclicity Criterion and has been used to determin the hypercyclicity of some classes of operators. The following version appears in [3].

Definition 1.1. Let $T \in L(X)$ and let $\left(n_{k}\right)$ be an increasing sequence of integers. We say that $T$ satisfies the Hypercyclicity Criterion for $\left(n_{k}\right)$ if there exist, two dense sets $Y, Z \subseteq X$ and a sequence of maps $S_{k}: Y \rightarrow X$ such that:

(i) $T^{n_{k}} y \rightarrow 0$ for all $y \in Y$,

Received March 17, 2011.

2010 Mathematics Subject Classification. Primary 47A16; Secondary 47B38.

Key words and phrases. hypercyclic operator, hereditarily criterion, mixing operator.

(C)2012 The Korean Mathematical Society 
(ii) $S_{k} z \rightarrow 0$ and $T^{n_{k}} S_{k} z \rightarrow z$ for all $z \in Z$.

We will say that $T$ satisfies the Hypercyclicity Criterion if it satisfies the criterion for some increasing sequence $\left(n_{k}\right)$ of positive integer. We point out that the Kitai Criterion has been given with the strong assumptions:

Definition 1.2. An operator $T \in L(X)$ is said to be satisfied the Kitai Criterion if there exist two dense subsets $Y$ and $Z$ of $X$ and a map $S: Z \rightarrow Z$ such that:

(i) $T^{n_{k}} y \rightarrow 0$ for all $y \in Y$,

(ii) $S^{k} z \rightarrow 0$ for all $z \in Z$,

(iii) $T S=I_{Z}$, where $I_{Z}$ is the identity operator on $Z$.

However, in some texts the Kitai Criterion is stated as the Hypercyclicity Criterion for whole sequence of natural numbers $(k)$ (see [1], page 4). For this reason, in the present paper, by the Kitai Criterion, we mean the Hypercyclicity Criterion for $(k)$.

It is easy to check that if $T \in L(X)$ satisfies the Hypercyclicity Criterion, then $T$ is hypercyclic. Moreover, the operator $T \oplus T$ acting on $X \oplus X$ is also hypercyclic. It was shown by J. Bes and A. Peris [3] that the converse is also true: if $T \oplus T$ is hypercyclic, then $T$ satisfies the Hypercyclicity Criterion.

It had been an open problem for a long time $[1,3,14]$ to know whether every hypercyclic operator satisfies the Hypercyclicity Criterion, equivalently, is $T \oplus T$ hypercyclic whenever $T$ is? By a recent result of M. De La Rosa and C. Read [12], there exist hypercyclic Banach space operators which do not satisfy the Hypercyclicity Criterion. Also it is proved that such operators can be constructed on a large class of Banach spaces, including $c_{0}(\mathbb{N})$ or $\ell^{p}(\mathbb{N})[2]$.

Bes and Peris [3] introduced the notion of hereditarily hypercyclicity and proved that $T$ satisfies the Hypercyclicity Criterion if and only if $T$ is hereditarily hypercyclic and if and only if the direct sum $T \oplus T$ is hypercyclic.

Definition 1.3. An operator $T \in L(X)$ is called hereditarily hypercyclic with respect to $\left(n_{k}\right)$, provided for all subsequences $\left(n_{k_{j}}\right)$ of $\left(n_{k}\right)$; the sequence $\left\{T^{n_{k_{j}}}\right.$ : $j \geq 1\}$ is hypercyclic, namely, the set $\left\{T^{n_{k_{j}}} x: j \geq 1\right\}$ is dense in $X$ for some $x \in X$. Moreover, $T$ is called hereditarily hypercyclic if $T$ is hereditarily hypercyclic with respect to some positive integer sequence $\left(n_{k}\right)$. When $n_{k}=k$ we say that $T$ is mixing.

It can be easily seen that an operator satisfying the criterion for $\left(n_{k}\right)$ is not only hypercyclic but also hereditarily hypercyclic with respect to $\left(n_{k}\right)$. In particular, operators satisfying Kitai Criterion are mixing. It is a question of Shapiro [17] to know whether the converse is true. Grivaux [7], answering the question raised by Shapiro, proved that if $B$ is a weighted backward shift operator on $\ell^{p}$, that its weights are small enough, then the perturbation of the identity operator by $B$, i.e., $I+B$ is a mixing operator which does not satisfy the Kitai Criterion. In fact, the Propositions 2.4 and 2.5 in [7] show 
that $I+B$ does not satisfy the Hypercyclicity Criterion for $(k)$. This result also was extended to any separable infinite dimensional Banach space.

A strictly increasing sequence of positive integers $\left(n_{k}\right)$ is said to be syndetic if $\sup \left(n_{k+1}-n_{k}\right)<+\infty$. A bounded operator $T$ on $X$ is called syndetically hypercyclic if for any syndetic sequence of positive integers $\left(n_{k}\right)$, the sequence $\left\{T^{n_{k}}\right\}$ is hypercyclic [10]. It has been show that an operator $T$ is syndetically hypercyclic if and only if $T$ satisfies the Hypercyclicity Criterion, equivalently $T$ is hereditarily hypercyclic. It is natural to ask what can be concluded about operators satisfying the Hypercyclicity Criterion as well as hereditarily hypercyclic operators with respect to a syndetic sequence.

In the present paper, we show operators satisfying the Hypercyclic Criterion for a syndetic sequence must satisfy the Kitai Criterion. Similarly, operators which are hereditarily hypercyclic with respect to a syndetic sequence must be mixing. We also construct a bounded weighted shift operator on sequence space $\ell^{1}$, satisfying the Hypercyclicity Criterion but not satisfy the Kitai Criterion. In particular, a hereditarily hypercyclic weighted shift without being mixing will be obtained.

\section{Kitai criterion and syndetic sequence}

In what follows $X$ is a separable infinite dimensional Frechet space and $\left(n_{k}\right)$ is a sequence of increasing positive integers.

Recall that a sequence $\left(n_{k}\right)$ of positive integers is called syndetic when

$$
\sup _{k}\left(n_{k+1}-n_{k}\right)<+\infty
$$

We begin with the following simple observation:

Lemma 2.1. Suppose that $X_{1}, X_{2}, \ldots, X_{N}$ are separable Frechet spaces and $T_{i} \in L\left(X_{i}\right)$ for $i=1,2, \ldots, N$. For given sequence $\left(n_{k}\right)$, if each $T_{i}$ satisfies the Hypercyclicity Criterion for $\left(n_{k}\right)$ on $X_{i}$, then $T_{1} \oplus T_{2} \oplus \cdots \oplus T_{N}$ so does on $X_{1} \oplus X_{2} \oplus \cdots \oplus X_{N}$.

Now we ready to state the main theorem of this section:

Theorem 2.2. Suppose that T satisfies the Hypercyclicity Criterion for a syndetic sequence $\left(n_{k}\right)$. Then $T$ satisfies the Kitai Criterion.

Proof. Assume that $M=\sup _{k}\left(n_{k+1}-n_{k}\right)$. Then by taking $n_{0}=1$, we have

$$
\mathbb{N}=\bigcup_{k=0}^{+\infty}\left(\left[n_{k-1}, n_{k}\right] \cap \mathbb{N}\right)
$$

and thus there exists an increasing infinite sequence $\sigma: \mathbb{N} \rightarrow \mathbb{N}$ such that for any $k \in \mathbb{N}$ there exits some integer $j$ with $|j| \leq M-1$ and

$$
k=n_{\sigma(k)}+j \text {. }
$$


Now assume that $T$ satisfies the criterion for $\left(n_{k}\right)$, then for two dense subsets $Y, Z$ of $X$ and functions $S_{k}: Z \rightarrow X$, conditions (i), (ii) of the Hypercyclicity Criterion are satisfied. Let

$$
A_{j}=\left\{k \in \mathbb{N}: k=n_{\sigma(k)}-j\right\} \quad \text { and } \quad B_{j}=\left\{k \in \mathbb{N}: k=n_{\sigma(k)}+j\right\}
$$

for any $j$ with $|j| \leq M-1$. Note that $A_{j} \cap B_{j}=\emptyset$ for $j \neq 0$ and $A_{0}=B_{0}$. Without loss of generality, we can assume both of $A_{j}$ and $B_{j}$ for any $j$, are infinite. The subsequences of $\left(n_{k}\right)$ can be divided into $2 M-1$ subsequence of the forms $\left\{k: k \in A_{j}\right\}$ and $\left\{k: k \in B_{j}\right\}$. Let $\tilde{Y}=T^{M}(Y)$ and $\tilde{Z}=T^{M}(Z)$. Then both $\tilde{Y}$ and $\tilde{Z}$ are dense subsets of $X$. By (2.1) we find that for each $y \in Y:$

$$
T^{k}\left(T^{M} y\right)=\left\{\begin{array}{lll}
T^{M-j}\left(T^{m_{\sigma(k)}} y\right) & \text { if } \quad k \in A_{j} \text { for some } j \\
T^{M+j}\left(T^{m_{\sigma(k)}} y\right) & \text { if } \quad k \in B_{j} \text { for some } j
\end{array}\right.
$$

We also define functions $\tilde{S}_{k}: \tilde{Z} \rightarrow X$ by

$$
\tilde{S}_{k}\left(T^{M} z\right)=\left\{\begin{array}{lll}
T^{M+j} S_{\sigma(k)}(z) & \text { if } \quad k \in A_{j} \\
T^{M-j} S_{\sigma(k)}(z) & \text { if } \quad k \in B_{j} .
\end{array}\right.
$$

For each $z \in Z$, using (2.1) with the above relations to get:

$$
T^{k} \tilde{S}_{k}\left(T^{M} z\right)-T^{M} z=T^{M}\left(T^{n_{\sigma(k)}} S_{\sigma(k)}(z)-z\right) \quad\left(k \in A_{j} \cup B_{j}\right) .
$$

Since conditions (i) and (ii) of the Hypercyclicity Criterion are true for $\left(n_{k}\right)$,

$$
\left\{\begin{array}{l}
T^{n_{\sigma(k)}}(y) \rightarrow 0 \text { for all } y \in Y \\
S_{\sigma(k)}(z) \rightarrow 0 \text { for all } z \in Z \\
T^{n_{\sigma(k)}} S_{\sigma(k)}(z) \rightarrow z \text { for all } z \in Z
\end{array}\right.
$$

Now by the above equalities we conclude that

$$
\left\{\begin{array}{l}
\tilde{T}^{k} \rightarrow 0, \text { poinwise on } \tilde{Y} \\
\tilde{S}_{k} \rightarrow 0, \text { pointwise on } \tilde{Z} \\
T^{n_{k}} \tilde{S}_{k} \rightarrow I_{Z}, \text { pointwise on } \tilde{Z}
\end{array}\right.
$$

Therefore, $T$ satisfies the Hypercyclicity Criterion for $(k)$ and the proof is complete.

Recall that if the operator $T$ satisfies the Hypercyclicity Criterion for $\left(n_{k}\right)$, then it is hereditarily hypercyclic with respect to $\left(n_{k}\right)$. However, by a result of Bes and Peris [3], the converse is true for the weighted shift operators:

Theorem 2.3. Let the operator $T$ on $\ell^{1}$ be determined by $T\left(e_{n}\right)=w_{n} e_{n-1}$ for $n \geq 2$ and $T\left(e_{1}\right)=0$. Then the following statments are equivalent:

(i) $T$ satisfies the Hypercyclicity Criterion for $\left(n_{k}\right)$,

(ii) $T$ is hereditarily with respect to $\left(n_{k}\right)$,

(iii) $\lim \prod_{s=2}^{j+n_{k}} w_{s}=+\infty$ for all $j \in \mathbb{N}$. 
Proof. That (ii) and (iii) are equivalent is deduced from Proposition 3.3 in [3]. It is also easy to see (i) implies (ii). Thus it remains to prove (iii) implies (i). For this, suppose condition (iii) holds. Let $Y=Z:=\operatorname{span}\left\{e_{i}: i \in \mathbb{N}\right\}$. Then

$$
T^{n_{k}}\left(e_{i}\right)=w_{i} w_{i-1} \cdots w_{i-n_{k}+1} e_{i-n_{k}}
$$

for every $i \in \mathbb{N}$. Now define functions $S_{k}: Y \rightarrow Y$ by

$$
S_{k}\left(e_{i}\right)=\frac{1}{w_{i+1} w_{i+2} \cdots w_{i+n_{k}}} e_{i+n_{k}} .
$$

By the above relation we find that $T^{n_{k}} y \rightarrow 0, S_{k} z \rightarrow 0$ and $T^{n_{k}} S_{k} z \rightarrow z$ for every $y \in Y$ and $z \in Z$. So $T$ satisfies the criterion for $\left(n_{k}\right)$ and the proof is completed.

Example 2.4. Every non-syndetic sequence $\left(n_{k}\right)$ induces a unilateral weighted shift on $\ell^{2}$ satisfying the Hypercyclicity Criterion for $\left(n_{k}\right)$, not satisfying the Kitai Criterion.

Proof. Since $\left(n_{k}\right)$ is not syndetic, we can find two increasing sequences $\left(p_{k}\right)$, $\left(q_{k}\right) \subseteq \mathbb{N}$ such that:

(i) $q_{k}<n_{p_{k}}<q_{k+1}$,

(ii) $\left|q_{k}-n_{p}\right|>k$ for each $p \geq 1$

(For example, if $n_{k}=(2 k)^{2}$, then $p_{k}=k$ and $\left.q_{k}=(2 k-1)^{2}\right)$.

Hence,

$$
n_{p_{k-1}}<q_{k}-k<q_{k}<q_{k}+k<n_{p_{k}} .
$$

Define the weighted sequence $w=\left\{w_{s}\right\}$ by:

$$
w_{s}=\left\{\begin{array}{lll}
2^{-1} & \text { if } q_{k}-k+1 \leq s \leq q_{k} & \text { for } \quad k>1 \\
2 & \text { if } q_{k}+1 \leq s \leq q_{k}+k & \text { for } \quad k>1 \\
1 & \text { otherwise }
\end{array}\right.
$$

By induction, we prove that $\prod_{s=1}^{q_{k}} w_{s}=1$. To this end, note that $\prod_{s=1}^{q_{1}} w_{s}=$ 1. Now suppose that $\prod_{s=1}^{q_{k-1}} w_{s}=1$ for $k>2$, we compute $\prod_{s=1}^{q_{k}} w_{s}$. Let $w(s)=w_{s}$, by using of

$$
1<q_{k-1}<q_{k-1}+1<q_{k-1}+k-1<q_{k-1}+k<q_{k}-k<q_{k}-k+1<q_{k},
$$

we split and then compute the product $\prod_{s=1}^{q_{k}} w_{s}$ as the following:

$$
\begin{aligned}
\prod_{s=1}^{q_{k}} w_{s}= & \left(w(1) w(2) \cdots w\left(q_{k}\right)\right) \\
= & \left(w(1) w(2) \cdots w\left(q_{k-1}\right)\right. \\
& \cdot\left(w\left(q_{k-1}+1\right) \cdots w\left(q_{k-1}+k-1\right)\right) \\
& \cdot\left(w\left(q_{k-1}+k\right) \cdots w\left(q_{k}-k\right)\right) \\
& \cdot\left(w\left(q_{k}-k+1\right) \cdots w\left(q_{k}\right)\right) \\
= & 1 \cdot 2^{k-1} \cdot 1 \cdot \frac{1}{2^{k-1}}=1 .
\end{aligned}
$$


In view of the preceding theorem, the unilateral weighted shift $T_{w}$ defined by the weighted sequence $w=\left\{w_{s}\right\}$, does not satisfy the Kitai Criterion. Now we prove $T_{w}$ satisfies the Hypercyclicity Criterion for $\left(n_{p}\right)$ on $\ell^{1}$. Let $p \geq 1$ and $n_{p_{k-1}}<n_{p}<n_{p_{k}}$. Using (i) and (ii), to get

$$
n_{p_{k-1}}<q_{k}, n_{p}<n_{p_{k}} \text { and }\left|n_{p}-q_{k}\right|>k \text {. }
$$

Thus, we have two possibilities:

Case (i): If $n_{p_{k-1}}<q_{k}<n_{p}<n_{p_{k}}$, then $n_{p}-q_{k}>k$ and hence

$$
\begin{aligned}
\prod_{s=1}^{n_{p}+j} w_{s}= & \left(w(1) \cdots w\left(n_{p}+j\right)\right) \\
= & \left(w(1) \cdots w\left(q_{k}\right)\right) \\
& \cdot\left(w\left(q_{k}+1\right) \cdots w\left(q_{k}+k\right)\right) \\
& \cdot\left(w\left(q_{k}+k+1\right) \cdots w\left(n_{p}\right)\right) \\
& \cdot\left(w\left(n_{p}+1\right) \cdots w\left(n_{p}+j\right)\right) \\
\geq & 1 \cdot \alpha^{k} \cdot 1 \cdot \alpha^{-j}=\alpha^{k-j} .
\end{aligned}
$$

Case (ii): If $n_{p_{k-1}} \leq n_{p}<q_{k}<n_{p_{k}}$, then $q_{k}-n_{p}>k$ and in this case

$$
\begin{aligned}
\prod_{s=1}^{n_{p}+j} w_{s}= & \left(w(1) \cdots w\left(n_{p}+j\right)\right) \\
= & \left(w(1) \cdots w\left(q_{k-1}\right)\right) \\
& \cdot\left(w\left(q_{k-1}+1\right) \cdots w\left(q_{k-1}+k-1\right)\right) \\
& \cdot\left(w\left(q_{k-1}+k\right) \cdots w\left(n_{p}\right)\right) \\
& \cdot\left(w\left(n_{p}+1\right) \cdots w\left(n_{p}+j\right)\right) \\
\geq & 1 \cdot \alpha^{k-1} \cdot 1 \cdot \alpha^{-j} \geq \alpha^{k-j-1}
\end{aligned}
$$

(In both cases, $\left(w\left(n_{p}+1\right) \cdots w\left(n_{p}+j\right)\right) \geq \alpha^{-j}$ because $\left.w_{s} \geq \alpha^{-1}\right)$. Now for each $p \geq n_{1}$, pick $k$ sufficiently large to satisfying $n_{p_{k-1}}<n_{p}<n_{p_{k}}$. Now from the above relations we conclude that if $p \rightarrow+\infty$, then $k \rightarrow+\infty$ and $\prod_{s=1}^{n_{p}+j} w_{s} \rightarrow \infty$.

\section{Mixing operators and syndetic sequence}

In what follows $X$ is a separable infinite dimensional Frechet space and $\left(n_{k}\right)$ is a sequence of increasing positive integers.

It is well known that the operator $T$ is hypercyclic if and only if for any given two open sets $U, V$ there is some positive integer $n$ such that $T^{n}(U) \cap V$ is nonempty (see Theorem 1.2 in [8]). A hereditarily hypercyclic operator satisfies a much stronger condition: 
Proposition 3.1. The operator $T \in L(X)$ is hereditarily hypercyclic with respect to $\left(n_{k}\right)$ if and only if for any pair $(U, V)$ of nonempty open subsets of $X$, there is some positive integer $N$ such that

$$
T^{n_{k}} U \cap V \neq \emptyset \text { for all } k>N \text {. }
$$

Proof. Without loss of generality suppose that $n_{k}=k$. For the proof of the sufficiency by way of contradiction, suppose that for a pair $(U, V)$ of nonempty open subsets of $X, T^{n_{k}} U \cap V=\emptyset$ for some subsequence $\left(n_{k}\right)$ of $(k)$. By definition, for a vector $x \in X$, the set $\left\{T^{n_{k}} x: k \geq 1\right\}$ is dense in $X$ whence $T^{i} x \in U$ for some integer $i$. Since $T$ has dense rang, the set $\left\{T^{n_{k}}\left(T^{i} x\right): k \geq 1\right\}$ is also dense in $X$ and consequently $T^{n_{k}}\left(T^{i} x\right) \in V$ for some $k$ large enough, a contradiction. For the prove the necessity fix a countable basis $\left\{V_{i}\right\}$ for the topology of $X$ and a subsequence $\left(n_{k}\right)$ of $(k)$. By assumption, each of the open sets $\bigcup_{k} T^{-n_{k}} V_{i}$ is dense in $X$ for any integer $i \geq 0$ and so is $\bigcap_{i} \bigcup_{k} T^{-n_{k}} V_{i}$. It is east to check that for any vector $x$ in the recent set, $\left\{T^{n_{k}} x: k \geq 1\right\}$ is dense in $X$. This completes the proof.

Corollary 3.2. The operator $T$ is mixing if and only if it is hereditarily hypercyclic with respect to the whole sequence $(n)$ of integers.

Lemma 3.3. Suppose that $X_{1}, X_{2}, \ldots, X_{N}$ are separable Banach spaces and $T_{i} \in B\left(X_{i}\right)$ for $i=1,2, \ldots, N$. For given $\left(n_{k}\right) \subseteq \mathbb{N}$, if $T_{i}$ is hereditarily hypercyclic with respect to $\left(n_{k}\right)$ on $X_{i}$, then $T_{1} \oplus T_{2} \oplus \cdots \oplus T_{N}$ dose so on $X_{1} \oplus X_{2} \oplus \cdots \oplus X_{N}$.

Theorem 3.4. Assume that $T$ is hereditarily hypercyclic with respect to a syndetic sequence $\left(n_{k}\right)$. Then $T$ is mixing.

Proof. Assume that $M=\sup _{k}\left|n_{k}-n_{k+1}\right|$. Then by taking $n_{0}=1$, we have

$$
\mathbb{N}=\bigcup_{k=0}^{+\infty}\left(\left[n_{k-1}, n_{k}\right] \cap \mathbb{N}\right)
$$

and thus there exists an increasing infinite sequence $\sigma: \mathbb{N} \rightarrow \mathbb{N}$ such that for any $k$ there exits some integer $j_{k}$ with $\left|j_{k}\right| \leq M-1$ and

$$
k=n_{\sigma(k)}+j_{k} .
$$

Now suppose $T$ is hereditarily hypercyclic with respect to $\left(n_{k}\right)$. Fix two open subsets $U, V$ in $X$. By Proposition 3.1, it suffices to show that $T^{k} U \cap V$ is nonempty for sufficiently large $k$. To do this, let $\hat{X}=X \oplus X \oplus \cdots \oplus X$ and $\hat{T}=T \oplus T \oplus \cdots \oplus T$, be $2 M-1$ copies of $X$ and $T$, respectively. By Lemma $2.1, \hat{T}$ is also hereditarily hypercyclic with respect to $\left(n_{k}\right)$ on $\hat{X}$. Consider the open subsets $\hat{U}, \hat{V}$ of $\hat{X}$ as the following:

$$
\hat{U}:=\underbrace{U \oplus U \oplus \cdots \oplus U}_{M \text { copies }} \oplus T^{-1} U \oplus \cdots \oplus T^{-M+2} U \oplus T^{-M+1} U \text { and }
$$




$$
\hat{V} \quad:=T^{-M+1} V \oplus T^{-M+2} V \oplus \cdots \oplus T^{-1} V \oplus \underbrace{V \oplus V \cdots \oplus V}_{M \text { copies }} .
$$

By Proposition 3.1, $\hat{T}^{n_{k}} \hat{U} \cap \hat{V} \neq \emptyset$ for sufficiently large $k$. In particular, for some positive integer $N, \hat{T}^{n_{\sigma(k)}} \hat{U} \cap \hat{V} \neq \emptyset$ for any $k>N$. The definitions of $\hat{T}, \hat{U}$ and $\hat{V}$ imply that for each $k>N$, there exists some integer $j_{k}$ with $\left|j_{k}\right| \leq M-1$, satisfying

$$
T^{n_{\sigma(k)}+j_{k}}(U) \cap V \neq \emptyset .
$$

We conclude from (1) and (2) that $T^{k} U \cap V \neq \emptyset$ for any $k>N$. Therefore by Corollary $3.2, T$ is mixing.

It must be mentioned that the operator in Example 2.4 is in fact a hereditarily hypercyclic operator which is not mixing.

\section{References}

[1] F. Bayart and E. Matheron, Daynamics of Linear Operators, Cambridge University Press, Cambridge, 2009.

$[2]$ - Hypercyclic operators failing the hypercyclicity criterion on classical Banach spaces, J. Funct. Anal. 250 (2007), no. 2, 426-441.

[3] J. Bes and A. Peris, Hereditarily hypercyclic operators, J. Funct. Anal. 167 (1999), no. $1,94-112$

[4] P. S. Bourdon and J. H. Shapiro, Cyclic phenomena for composition operators, Mem. Amer. Math. Soc. 125 (1997), no. 596, x+105 pp.

[5] K. C. Chan and J. H. Shapiro, The cyclic behavior of translation operators on Hilbert spaces of entire functions, Indiana Univ. Math. J. 40 (1991), no. 4, 1421-1449.

[6] R. M. Gethner and J. H. Shapiro, Universal vectors for operators on spaces of holomorphic functions, Proc. Amer. Math. Soc. 100 (1987), no. 2, 281-288.

[7] S. Grivaux, Hypercyclic operators, mixing operators, and the bounded steps problem, J. Operator Theory 54 (2005), no. 1, 147-168.

[8] G. Godefroy and J. H. Shapiro, Operators with dense invariant cyclic vector manifolds, J. Funct. Anal. 98 (1991), no. 2, 229-269.

[9] C. Kitai, Invariant closed sets for linear operators, Ph.D. thesis, Univ. of Toronto, Toronto, 1982.

[10] A. Peris and L. Salvidia, Syndetically hypercyclic operators, Integral Equations Operator Theory 51 (2005), no. 2, 275-281.

[11] H. Rezaei, Notes on dynamics of the adjoint of a weighted composition operator, Taiwanese J. Math. 14 (2010), no. 4, 1377-1384.

[12] S. Rolewicz, On orbits elements, Studia Math. 32 (1969), 17-22.

[13] M. de la Rosa and C. Read, A hypercyclic operator whose direct sum is not hypercyclic, preprint, 2006.

[14] F. Leon-Saavedra and A. Montes-Rodryguez, Linear structure of hypercyclic vectors, J. Funct. Anal. 148 (1997), no. 2, 524-545.

[15] H. Salas, Hypercyclic weighted shifts, Trans. Amer. Math. Soc. 347 (1995), no. 3, 9931004.

[16] J. H. Shapiro, Notes on the dynamics of linear operators, unpublished notes.

[17] B. Yousefi and H. Rezaei, Hypercyclic property of weighted composition operators, Proc. Amer. Math. Soc. 135 (2007), no. 10, 3263-3271. 
Department of Mathematics

College of Sciences

YASOUJ UNIVERSITY

YASOUJ, 75918-74831, IRAN

E-mail address: rezaei@mail.yu.ac.ir 